\title{
Metabolic Syndrome and Cognitive Decline in Elderly Latinos: Findings from the Sacramento Area Latino Study of Aging Study
}

\author{
Kristine Yaffe, MD, ${ }^{* \neq}{ }^{*} \|$ Mary Haan, DrPH, MPH, Terri Blackwell, MA, Elena Cherkasova, BA, ${ }^{\mathcal{S}}$ \\ Rachel A. Whitmer, PhD, ${ }^{* *}$ and Nancy West, $M S^{*}$
}

OBJECTIVES: To investigate the effect of metabolic syndrome on cognitive function in an elderly Latino population and to determine whether inflammation modifies this association.

DESIGN: A longitudinal cohort study.

SETTING: Sacramento area and the surrounding California counties from 1998 to 1999.

PARTICIPANTS: One thousand six hundred twenty-four Latinos aged 60 and older who participated in the Sacramento Area Latino Study of Aging.

MEASUREMENTS: Baseline metabolic syndrome was calculated using the Third Adult Treatment Panel of the National Cholesterol Education Program. Cognitive function was measured using the Modified Mini-Mental State Examination (3MS) and the Delayed Word-List Recall (DelRec), a verbal memory test. The effect of metabolic syndrome on cognitive change scores was examined using random effects models; in addition, the effect of the individual components of the syndrome on cognitive change was examined.

RESULTS: Of the 1,624 participants, 718 (44\%) had metabolic syndrome at baseline. Those with metabolic syndrome had worse 3 -year change scores on 3MS $(P=.04)$ and DelRec $(P=.03)$. Multivariate adjustment attenuated the results for DelRec but not for 3MS. This association was especially pronounced in participants with a high serum level of inflammation, resulting in an average $3 \mathrm{MS}$ score 0.64 points lower per year $(P=.03)$ for those with metabolic syndrome. Individual components of metabolic syndrome were not associated with cognitive decline except for elevated glucose on the DelRec $(P=.02)$ and high blood pressure on $3 \mathrm{MS}(P=.05)$.

From the Departments of *Psychiatry, ${ }^{\dagger}$ Neurology, ${ }^{\ddagger}$ Epidemiology and Biostatistics, and ${ }^{\S}$ Geriatrics, University of California at San Francisco, San Francisco, California; "San Francisco VA Medical Center, San Francisco, California; "School of Public Health, University of Michigan, Ann Arbor,

Michigan; ${ }^{\#}$ California Pacific Medical Center, San Francisco, California; and

** Division of Research, Kaiser Permanente, Oakland, California.

Address correspondence to Kristine Yaffe, MD, c/o University of California, San Francisco, Box 181, 4150 Clement Street, San Francisco, CA 94121.

E-mail: Kristine.yaffe@ucsf.edu

DOI: $10.1111 / \mathrm{j} .1532-5415.2007 .01139 . x$
CONCLUSION: Metabolic syndrome and inflammation may both contribute to cognitive decline in older people of diverse backgrounds. The results also suggest that, in elderly Latinos, the composite measure of metabolic syndrome is a greater risk for cognitive decline than its individual components. J Am Geriatr Soc 55:758-762, 2007.

Key words: metabolic syndrome; cognition; dementia; diabetes mellitus; elderly

$M$ etabolic syndrome is a constellation of cardiovascular risk factors that include abdominal obesity, hypertriglyceridemia, low high-density lipoprotein (HDL) levels, hypertension, and hyperglycemia ${ }^{1}$ and is associated with greater risk of developing diabetes mellitus and cardiovascular disease. Although several of these cardiovascular and metabolic risk factors have been independently associated with a greater risk of developing dementia or cognitive decline, the effect of metabolic syndrome on cognitive function has not been extensively studied. It was recently demonstrated that metabolic syndrome contributes to cognitive impairment in older black and white people, particularly in those with high levels of inflammation. ${ }^{2}$

By 2005, Hispanic Americans are estimated to become the largest ethnic minority group in the United States. ${ }^{3}$ This is especially significant because older Hispanic people have a high prevalence of diabetes mellitus and metabolic syndrome, ${ }^{4}$ although few studies have examined the effects of this higher prevalence on cognitive outcomes. The present study was conducted to investigate the effect of metabolic syndrome on cognitive function in an elderly Hispanic population and to determine whether inflammation modified this association. As part of an ongoing prospective study, whether metabolic syndrome was associated with cognitive decline in older people without dementia was determined.

The Sacramento Area Latino Study of Aging (SALSA) study was an ideal cohort in which to address this question, because the participants were all older community-dwelling Latinos who had careful characterization of metabolic 
syndrome and inflammation and were prospectively followed with cognitive assessments. The hypothesis was that presence of metabolic syndrome would be associated with more-rapid cognitive decline and that inflammation would modify this association.

\section{METHODS}

\section{Subjects}

SALSA participants were recruited from the Sacramento area and the surrounding California counties from 1998 to 1999. A detailed description of the study and recruitment has been described elsewhere. ${ }^{4}$ In brief, participants were eligible for enrollment if they were aged 60 and older and self-designated as Latino. Bilingual technicians performed annual interviews in the primary language of the participant at the participant's home. Of the 1,789 participants initially enrolled, 1,624 with data on metabolic syndrome and cognitive function constituted the analytical cohort. This analysis covers the period from baseline to the third annual follow-up visit.

The institutional review boards of the University of California at Davis and the University of California at San Francisco approved the study, and written informed consent was obtained from all participants.

\section{Measurements}

Baseline metabolic syndrome was calculated using the Third Adult Treatment Panel of the National Cholesterol Education Program. ${ }^{1}$ Participants were considered to have metabolic syndrome if they met three or more of five criteria: abdominal obesity (waist circumference $>88 \mathrm{~cm}$ for women; $>102 \mathrm{~cm}$ for men), hypertriglyceridemia $(\geq 150 \mathrm{mg} / \mathrm{dL}){ }^{3}$ low HDL cholesterol $(<40 \mathrm{mg} / \mathrm{dL}$ for men; $<50 \mathrm{mg} / \mathrm{dL}$ for women), high blood pressure (systolic $\geq 130 \mathrm{mmHg}$, diastolic $\geq 85 \mathrm{mmHg}$ ) or use of antihypertensive medication, and high fasting blood glucose $(\geq 110 \mathrm{mg} /$ $\mathrm{dL}$ ) or use of antidiabetic medication.

At baseline, fasting blood was collected from each participant and stored at $-70^{\circ} \mathrm{C}$ at the University of California at Davis. High-sensitivity C-reactive protein (CRP) was analyzed at the University of Michigan CLASS laboratory using the Equal Diagnostics high-sensitivity CRP kit (Exton, PA). Inflammation level was dichotomized at the median level $(3.2 \mathrm{mg} / \mathrm{L})$, which is similar to the American Heart Association and Centers for Disease Control and Prevention recommended cutoff of $3.0 \mathrm{mg} / \mathrm{L}$.

Cognitive function was measured using the Modified Mini-Mental State Examination (3MS) and the Delayed Word-List Recall (DelRec) from the Spanish-English verbal learning test. These measurements were collected at baseline and each year postbaseline. The $3 \mathrm{MS}$ is a global measure of cognitive functioning, with components for orientation, concentration, language, praxis, and immediate and delayed recall; scores range from 0 to 100 , with higher scores representing better cognition. ${ }^{5}$ The DelRec is a test of short-term verbal memory; scores range from 0 to 15 , with higher scores representing better functioning. ${ }^{6}$ Both tests were validated in Spanish and English.

At baseline, information was gathered on participants' age, sex, education (years), primary language, country of birth, and current smoking and alcohol use. Report of a physician diagnosis of stroke or myocardial infarction (MI) was gathered from the participant at each visit. Depressive symptoms were assessed using the Center for Epidemiologic Studies Depression Scale. ${ }^{7}$ The standard of 16 or more depressive symptoms was used to define presence of depression. The Acculturation Rating Scale for Latinos was used to measure acculturation. ${ }^{8}$

\section{Statistical Analyses}

Comparisons of baseline characteristics of those with and without metabolic syndrome were performed using $t$ tests for normally distributed continuous variables, Wilcoxon rank-sum tests for those with skewed distributions, and chisquare tests for categorical data. Transformations on both cognitive tests were necessary to meet the normality assumption of the models used. The DelRec was transformed using - (DelRec Score +1$)$; the 3MS was transformed using $-\log (-\log ((3 \mathrm{MS}$ score $) / 101+0.001))$. All results were backtransformed for presentation.

Random-effects models were used to analyze the association between the presence of metabolic syndrome at baseline and the 3-year change in cognitive functioning as measured using the 3MS and the DelRec. These models account for between-participant variation and within-participant correlation of repeated outcomes. ${ }^{9}$ The randomeffect terms included the intercept and the slope of the cognitive measurements over time, allowing for individual time trends for each participant. Variances and covariances were estimated using the restricted maximum likelihood method. Time was modeled as a continuous covariate, measured as years from baseline. The covariates (fixed effects) were determined using backward selection with a $P<.10$ selection criterion. If a variable was selected for the $3 \mathrm{MS}$ or the DelRec model, it was kept in both models, for consistency. The results presented are beta coefficients and $P$-values for the predictor, which represent the baseline difference between mean cognitive scores of those with metabolic syndrome and those without, and the beta coefficients and $P$-values for the interaction term of time and the predictor, which represents the difference over time in mean cognitive scores between those with metabolic syndrome and those without.

Similar analyses were performed using the five individual components of metabolic syndrome as predictors, with separate models for each component to assess whether the effect of the individual components differed from the effect of metabolic syndrome. Analyses were also performed after stratifying participants according to their CRP level $(\geq 3.2$ vs $<3.2 \mathrm{mg} / \mathrm{L}$ ). Analyses were preformed using SAS (version 9.1, SAS Institute, Inc., Cary, NC).

\section{RESULTS}

The mean age \pm standard deviation of the whole cohort was $70.5 \pm 7.0$. Of the 1,624 participants, $718(44 \%)$ had metabolic syndrome at baseline. Of those, 593 (83\%) had abdominal obesity, $573(80 \%)$ had hypertriglyceridemia, $419(58 \%)$ had low HDL cholesterol, 164 (23\%) had high blood pressure or were taking antihypertensive medication, and $473(66 \%)$ had high fasting blood glucose or were taking antidiabetic medication. Those with metabolic syndrome were more likely to be female, to have been born in 
Table 1. Baseline Characteristics of Sacramento Area Latino Study of Aging Participants with and without Metabolic Syndrome

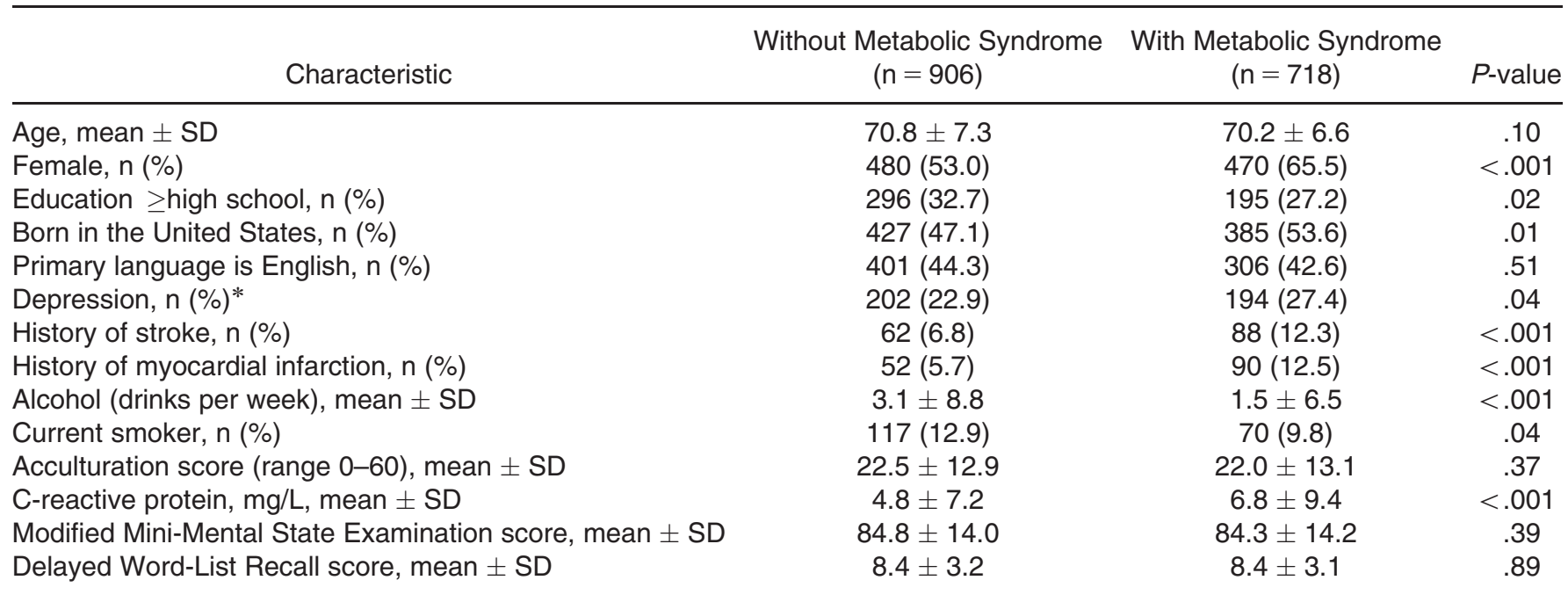

* Center for Epidemiological Studies Depression Scale score $\geq 16$.

$\mathrm{SD}=$ standard deviation

the United States, to be depressed, and to have a history of stroke or MI than those without metabolic syndrome $(P<.05)$ (Table 1). Those with metabolic syndrome were less likely to be high school educated or to be current smokers $(P=.4)$ and consumed less alcohol $(P<.05)$. There was no significant difference in age, primary language, or acculturation between the two groups. As expected, participants with metabolic syndrome had higher mean levels of CRP than those without the syndrome $(P<.001)$.

Mean baseline cognitive function was $84.6 \pm 14.1$ using the 3MS and $8.4 \pm 3.2$ using the DelRec. The average follow-up time was $2.6 \pm 1.3$ years, with $65 \%$ of the analysis population having cognitive data gathered through Year 3. The reasons for lack of follow-up include death $(\mathrm{n}=120)$, refusal $(\mathrm{n}=142)$, lost to follow-up $(\mathrm{n}=137)$, had follow-up but no cognitive testing ( $\mathrm{n}=77$ ), and still followed but did not have testing within the timeframe of the return visit $(n=91)$. The proportion of participants with baseline presence of metabolic syndrome or elevated CRP level did not differ for those with and without followup. For the entire cohort, the repeated-measures model indicated no significant difference in baseline cognitive scores for those with and without metabolic syndrome for the 3MS or the DelRec (Tables 1 and 2), although those with metabolic syndrome had greater decline on the 3MS $(P=.04)$ and on the DelRec $(P=.03)$ (Table 2$)$. After multivariate adjustment for age, sex, education, place of birth, depression, history of stroke, MI, smoking, and alcohol use, those with metabolic syndrome scored 0.39 points lower per year on the $3 \mathrm{MS}$ than those without metabolic

Table 2. Association Between Metabolic Syndrome and Cognitive Change: Unadjusted and Multivariate-Adjusted Random-Effects Model

\begin{tabular}{|c|c|c|c|c|c|c|c|c|}
\hline \multirow[b]{3}{*}{ Model } & \multicolumn{4}{|c|}{ Modified Mini-Mental State Examination Score } & \multicolumn{4}{|c|}{ Delayed Word-List Recall Score } \\
\hline & \multicolumn{2}{|c|}{ Metabolic Syndrome } & \multicolumn{2}{|c|}{$\begin{array}{c}\text { Metabolic Syndrome } \\
\text { by Time }\end{array}$} & \multicolumn{2}{|c|}{ Metabolic Syndrome } & \multicolumn{2}{|c|}{$\begin{array}{c}\text { Metabolic Syndrome } \\
\text { by Time }\end{array}$} \\
\hline & Beta & $P$-value & Beta & $P$-value & Beta & $P$-value & Beta & $P$-value \\
\hline \multicolumn{9}{|l|}{ Unadjusted } \\
\hline All & -0.41 & .44 & -0.35 & .04 & 0.07 & .67 & -0.12 & .03 \\
\hline $\mathrm{CRP} \geq$ median & -0.23 & .78 & -0.46 & .04 & -0.18 & .51 & -0.13 & .16 \\
\hline $\mathrm{CRP}<$ median & -0.69 & .41 & -0.20 & .45 & -0.13 & .65 & -0.06 & .45 \\
\hline \multicolumn{9}{|c|}{ Multivariate adjusted* } \\
\hline All & -0.29 & .56 & -0.39 & .04 & -0.12 & .38 & -0.07 & .17 \\
\hline $\mathrm{CRP} \geq$ median & 0.43 & .61 & -0.64 & .03 & -0.25 & .26 & -0.07 & .33 \\
\hline $\mathrm{CRP}<$ median & -1.07 & .20 & -0.25 & .44 & -0.32 & .17 & -0.02 & .80 \\
\hline
\end{tabular}

* Multivariate models adjusted for age, sex, education, born in the United States, depression, history of stroke, history of myocardial infarction, smoking, alcohol, and missing pattern indicator.

$\mathrm{CRP}=$ C-reactive protein. 
Table 3. Association Between the Individual Components of Metabolic Syndrome and Cognitive Scores: MultivariateAdjusted Random-Effects Model

\begin{tabular}{|c|c|c|c|c|c|c|c|c|}
\hline \multirow[b]{3}{*}{ Component } & \multicolumn{4}{|c|}{$\begin{array}{l}\text { Modified Mini-Mental State } \\
\text { Examination Score }\end{array}$} & \multicolumn{4}{|c|}{ Delayed Word-List Recall Score } \\
\hline & \multicolumn{2}{|c|}{ Component } & \multicolumn{2}{|c|}{$\begin{array}{l}\text { Component } \\
\text { by Time }\end{array}$} & \multicolumn{2}{|c|}{ Component } & \multicolumn{2}{|c|}{$\begin{array}{l}\text { Component } \\
\text { by Time }\end{array}$} \\
\hline & Beta & $P$-value & Beta & $P$-value & Beta & $P$-value & Beta & $P$-value \\
\hline Abdominal obesity & -0.31 & .55 & -0.32 & .11 & 0.02 & .90 & -0.06 & .25 \\
\hline Hypertriglyceridemia & 0.20 & .69 & 0.03 & .87 & 0.27 & .05 & -0.05 & .34 \\
\hline Low high-density lipoprotein cholesterol & -0.32 & .54 & -0.33 & .11 & -0.15 & .32 & -0.01 & .87 \\
\hline High blood pressure & -0.01 & .98 & -0.37 & .05 & -0.17 & .21 & -0.03 & .54 \\
\hline High fasting blood glucose & -1.73 & $<.001$ & -0.31 & .10 & -0.19 & .18 & -0.12 & .02 \\
\hline
\end{tabular}

Note: Mulitvariate models adjusted for time, age, sex, education level, born in the United States, depression, history of stroke, history of myocardial infarction, smoking, alcohol, and missing pattern indicator.

syndrome $(P=.04)$, but the difference between the two groups on the DelRec score was no longer statistically significant $(P=.17)$.

Participants with metabolic syndrome with high inflammation (median CRP level $\geq 3.2 \mathrm{mg} / \mathrm{L}$ ) had an even greater multivariate-adjusted decline in $3 \mathrm{MS}$ score than those without high inflammation, with an average score 0.64 lower points per year $(P=.03)$. After stratification according to level of inflammation, there were no significant differences between those with and without metabolic syndrome on the DelRec (Table 2).

The five individual components of metabolic syndrome were also examined as predictors of cognitive change. At baseline, participants with elevated fasting blood glucose or use of antidiabetic medications had lower multivariate adjusted scores on the 3MS $(P<.001)$, and those with elevated triglyceride levels had lower scores on the DelRec $(P=.05)$ (Table 3). Only elevated fasting blood glucose or use of antidiabetic medications was statistically significantly associated with a lower score on DelRec over time $(P \leq .02)$. There was a trend-level association with high blood pressure and lower score on the $3 \mathrm{MS}$ over time $(P=.05)$. There was no association between the other three components of metabolic syndrome and longitudinal cognitive change (Table 3), Further stratification according to CRP level did not appreciably change these results.

\section{DISCUSSION}

The rate of cognitive change, as measured using the 3MS, was worse in older Latinos with metabolic syndrome than in those without the syndrome. This effect was even more marked in those with elevated levels of serum markers of inflammation. These results provide further evidence that metabolic syndrome and inflammation play a role in cognitive aging.

Previous studies have investigated the individual components of metabolic syndrome and risk of cognitive decline or dementia. Mid- or late-life hypertension, ${ }^{10,11}$ hyperlipidemia, ${ }^{12,13}$ and diabetes mellitus ${ }^{14-16}$ have been reported to increase the risk of developing cognitive impairment due to vascular disease and Alzheimer's disease, but few have assessed the combined effect of multiple cardiovascular risk factors such as that represented in metabolic syndrome. The results of the current study suggest that, in elderly Latinos, the composite measure of metabolic syndrome is a greater risk for cognitive decline than its individual components, with the possible exception of impaired glucose control and high blood pressure.

After multivariate adjustment, the association between metabolic syndrome and cognitive decline was only evident for the 3MS, a measure of global cognitive function, and not for the DelRec, a test of verbal memory. The meaning of this is not clear but may suggest that the underlying mechanism for the association may be due to vascular disease and not Alzheimer's disease, in which verbal memory is classically affected. Because it was not possible to define the clinical etiology of cognitive decline, there was not an opportunity to determine whether there is a stronger association between metabolic syndrome and cognitive decline due to vascular disease than that due to Alzheimer's disease.

High levels of inflammation increase the risk of the development of diabetes mellitus and atherosclerosis and are thought to be a possible mechanism for the adverse consequences of metabolic syndrome. ${ }^{17,18}$ In addition, markers of inflammation have been associated with the risk of developing dementia and cognitive decline. ${ }^{19,20}$ It was found that, in elderly Latinos, those with metabolic syndrome and high inflammation, as measured according to CRP, had accelerated cognitive aging. This is consistent with work in black and white community-dwelling older people. ${ }^{2}$ The magnitude of the effect of metabolic syndrome on those with elevated serum markers of inflammation was similar in both studies. It may be that the presence of inflammation indicates disease severity of metabolic syndrome or that there is a combined effect of metabolic syndrome and inflammation on cognitive decline.

The strengths of this study include its population-based design, annual assessment of cognitive tests, and the careful measurement and subsequent adjustment of possible confounders. Several limitations are evident in the study, including lack of the ability to measure a wide variety of cognitive domains and to define underlying etiology of cognitive change. There was thus no opportunity to 
determine whether there is a stronger association between metabolic syndrome and cognitive decline due to vascular disease than that due to Alzheimer's disease or vice versa. Although demographics and several comorbidities, including depression, smoking, MI, and stroke, were adjusted for, it is possible that there is residual confounding. It is conceivable that older people with high CRP levels and metabolic syndrome may have worse health outcomes and that these in turn, contribute to cognitive decline. In addition, there was only one measure of inflammation, and it would have been more ideal to have other inflammatory markers and measured on more than one clinic visit.

This study is important given that risk factors for cognitive decline have been relatively uncharacterized in Hispanic populations. Understanding the health consequences of metabolic syndrome, especially in those with inflammation, is crucial in a population that carries a higher incidence and prevalence of this syndrome. Future studies are needed to examine whether modifications in these risk factors could lower the risk of developing cognitive decline or dementia.

\section{ACKNOWLEDGMENTS}

Financial Disclosure: None of the authors had any financial arrangements or conflicts with an organization or company at the time of submission.

Author Contributions: Kristine Yaffe: concept design, analysis and interpretation of data, preparation of manuscript. Mary Haan: acquisition of subjects, preparation of manuscript. Terri Blackwell, Elena Cherkasova, Rachel Whitmer, and Nancy West: analysis of data, preparation of manuscript.

Sponsor's Role: None.

\section{REFERENCES}

1. Executive Summary of the Third Report of the National Cholesterol Education Program (NCEP) Expert Panel on Detection, Evaluation, and Treatment of High Blood Cholesterol in Adults (Adult Treatment Panel III). JAMA 2001; 285:2486-2497.
2. Yaffe K, Kanaya A, Lindquist $\mathrm{K}$ et al. The metabolic syndrome, inflammation, and risk of cognitive decline. JAMA 2004;292:2237-2242.

3. U.S. Census Bureau. National Population Projections. Washington, DC: U.S. Government Printing Office, 2000.

4. Haan MN, Mungas DM, Gonzalez HM et al. Prevalence of dementia in older Latinos: The influence of type 2 diabetes mellitus, stroke and genetic factors. J Am Geriatr Soc 2003;51:169-177.

5. Teng EL, Chui HC. The Modified Mini-Mental State (3MS) examination. J Clin Psychiatry 1987;48:314-318.

6. Gonzalez HM, Mungas D, Reed BR et al. A new verbal learning and memory test for English- and Spanish-speaking older people. J Int Neuropsychol Soc 2001;7:544-555.

7. Radloff L. The CES-D Scale: A self-report depression scale for research in the general population. Appl Psychol Meas 1977;1:385-401.

8. Cuellar I, Arnold B, Maldonado R. Acculturation Rating Scale for Mexican Americans. II. A revision of the original ARSMA Scale. Hisp J Behav Sci 1995;17:274-304.

9. Laird NM, Ware JH. Random-effects models for longitudinal data. Biometrics 1982;38:963-974.

10. Launer LJ, Masaki K, Petrovitch $\mathrm{H}$ et al. The association between midlife blood pressure levels and late-life cognitive function. The Honolulu-Asia Aging Study. JAMA 1995;274:1846-1851.

11. Knopman D, Boland LL, Mosley T et al. Cardiovascular risk factors and cognitive decline in middle-aged adults. Neurology 2001;56:42-48.

12. Moroney JT, Tang MX, Berglund L et al. Low-density lipoprotein cholesterol and the risk of dementia with stroke. JAMA 1999;282:254-260.

13. Evans RM, Emsley CL, Gao S et al. Serum cholesterol, APOE genotype, and the risk of Alzheimer's disease: A population-based study of African Americans. Neurology 2000;54:240-242.

14. Wu JH, Haan MN, Liang J et al. Impact of diabetes on cognitive function among older Latinos: A population-based cohort study. J Clin Epidemiol 2003;56:686-693.

15. Yaffe K, Blackwell T, Kanaya AM et al. Diabetes, impaired fasting glucose, and development of cognitive impairment in older women. Neurology 2004;63: 658-663.

16. Grodstein F, Chen J, Wilson RS et al. Type 2 diabetes and cognitive function in community-dwelling elderly women. Diabetes Care 2001;24: 1060-1065.

17. Barzilay JI, Abraham L, Heckbert SR et al. The relation of markers of inflammation to the development of glucose disorders in the elderly. The Cardiovascular Health Study. Diabetes 2001;50:2384-2389.

18. Pradhan AD, Manson JE, Rifai N et al. C-reactive protein, interleukin 6, and risk of developing type 2 diabetes mellitus. JAMA 2001;286:327-334.

19. Yaffe K, Lindquist K, Penninx BW et al. Inflammatory markers and cognition in well-functioning African-American and white elders. Neurology 2003;61: 76-80.

20. McGeer EG, McGeer PL. Brain inflammation in Alzheimer disease and the therapeutic implications. Curr Pharm Des 1999;5:821-836. 